\title{
Presenilins Function as ER Calcium Leak Channels: Implications for Alzhiemer's Disease
}

\author{
I. Bezprozvanny \\ UT Southwestern Medical Center at Dallas, Dallas, TX 75390, USA; \\ e-mail: Ilya.Bezprozvanny@UTSouthwestern.edu
}

DOI: $10.1134 / \mathrm{S} 199074780903012 \mathrm{X}$

\begin{abstract}
Alzheimer's disease (AD) is a progressive and irreversible neurodegenerative disorder. Mutations in presenilins are responsible for approximately $40 \%$ of all early onset familial Alzheimer's disease (FAD) cases in which a genetic cause has been identified. FAD mutations and genetic deletions of presenilins have been linked with calcium signaling abnormalities, but mechanistic basis for these results has not been clearly determined. Presenilins are highly conserved transmembrane proteins that support cleavage of the amyloid precursor protein by gamma-secretase. In our studies we discovered that in addition to acting as a gamma-secretase, presenilins also function as passive endoplasmic reticulum $\mathrm{Ca}^{2+}$-leak channels. We demonstrate that wild type PS1 and PS2 proteins form low conductance divalent cation-permeable ion channels in planar lipid bilayers. In experiments with PS1/2 double knockout (DKO) mouse embryonic fibroblasts (MEFs) we discovered that presenilins account for $\sim 80 \%$ of passive $\mathrm{Ca}^{2+}$ leak from the endoplasmic reticulum (ER). The ER $\mathrm{Ca}^{2+}$-leak function of presenilins is independent from their gamma-secretase function. In additional experiments we demonstrated that $\mathrm{ER} \mathrm{Ca}^{2+}$-leak function of presenilins is impaired by many FAD-causing
\end{abstract}

mutations in presenilins. Our data uncover a novel $\mathrm{Ca}^{2+}$ signaling function of presenilins and provide support to the potential role of disturbed $\mathrm{Ca}^{2+}$ homeostasis in $\mathrm{AD}$ pathogenesis. Recently we expanded our studies of presenilins and $\mathrm{Ca}^{2+}$ signaling to neuronal system. Our latest findings will be discussed.

Supported by McKnight Neuroscience of Brain Disorders Award, Robert A. Welch Foundation, the Institute for the Study of Aging, the NINDS grants R01NS38082 and R01NS056224.

\section{REFERENCES}

1. Tu, H., Nelson, O., Bezprozvanny, A., Wang, Z., Lee, S.F., Hao, Y.H., Serneels, L., De Strooper, B., Yu, G., and Bezprozvanny, I., Presenilins form ER $\mathrm{Ca}^{2+}$ Leak Channels, a Function Disrupted by Familial Alzheimer's Disease-Linked Mutations, Cell, 2006, vol. 126, pp. 981-993.

2. Nelson, O., Tu, H., Lei, T., Bentahir, M., de Strooper, B., and Bezprozvanny, I., Familial Alzheimer DiseaseLinked Mutations Specifically Disrupt $\mathrm{Ca}^{2+}$ Leak Function of Presenilin 1. J. Clin. Investigation, 2007, vol. 117, pp. 1230-1239. 